\title{
A CLOSE LOOK AT THE OUTBURST OF THE VERY SLOW SYMBIOTIC NOVA PU VUL - A TRIPLE SYSTEM
}

\author{
D. CHOCHOL ${ }^{1}$, S. TAMURA ${ }^{2}$, O. KANAMITSU ${ }^{3}$, \\ T. PRIBULLA ${ }^{1}$, A. TAJITSU ${ }^{2}$ \\ 1. Astronomical Institute of the Slovak Academy of Sciences, \\ 05960 Tatranská Lomnica, Slovakia \\ 2. Astronomical Institute, Faculty of Science, Tohoku \\ University, Aoba-ku, Sendai, Miyagi 980, Japan \\ 3. Fukuoka University of Education, 799 Akama, \\ Munakata-shi, Fukuoka 811-41, Japan
}

The very slow nova PU Vul was discovered by Honda and Kuwano in 1979 after the rise from $B \sim 15 \ldots 16 \mathrm{mag}$ to a maximum $B \sim 9 \mathrm{mag}$. The object has been studied by many authors (for references see Belyakina et al. 1989; Vogel \& Nussbaumer 1992) but a clear picture of the system was not given. Two minima of different duration were observed in the system in the active stage, in 1980-81 and 1993-94 (Kolotilov et al. 1995). Nussbaumer \& Vogel (1996) ascribed the minima to eclipses of the hot component by the cool M giant in a binary with orbital period of $4900 \pm 100 \mathrm{~d}$. Our period analysis of the preoutburst $B$ data (Liller \& Liller 1979) by Stellingwerf's (1978) method revealed a period of $4858 \mathrm{~d}$. We found the third minimum in 1926-28 (lasting for $500 \ldots 630 \mathrm{~d}$ ) in these data. The centers of the three minima were used to improve the ephemeris:

$$
\text { JD }(\min .)=2425032( \pm 30)+4882( \pm 10) E \text {. }
$$

The different lengths of the eclipses, their asymmetry and their longer $\mathrm{du}$ ration than expected from the standard characteristics of the components in a binary, can all be explained by supposing that PU Vul is a hierarchical triple system. The observed minima are caused by the eclipses of the white dwarf in the inner orbit by the $M$ giant in the outer orbit. The parameters of the inner orbit found from the radial velocity curve of the white dwarf, mimicking an F supergiant from 1979 to 1987 (for references see Kanamitsu et al. 1991), are given in Fig. 1. The spectroscopic orbital period of $761 \mathrm{~d}$ is close to the most significant period found in $B(768 \mathrm{~d})$ and $V(782 \mathrm{~d})$ photometric data obtained in the years 1982-87. Analysis of the $U$ data revealed the most significant period of $328 \mathrm{~d}$, which corresponds to the mass transfer bursts from the inner $\mathrm{M}$ giant onto the hot component. 


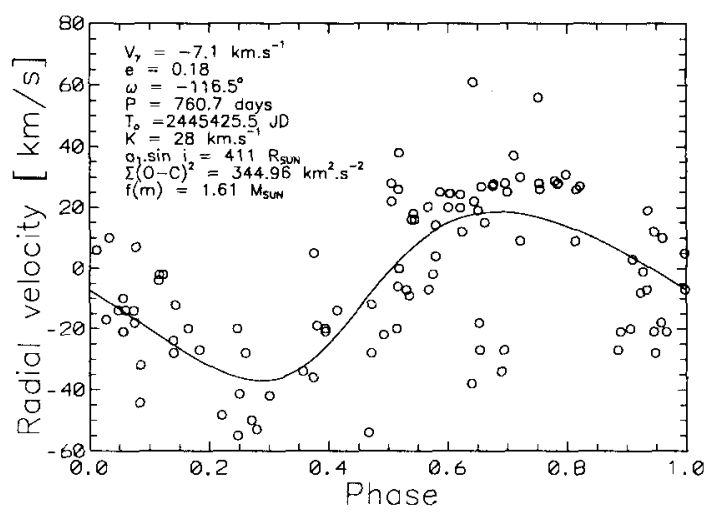

Figure 1. Spectroscopic orbit of the slow symbiotic nova PU Vul

An expanding atmosphere of the hot star formed a common envelope surrounding the inner binary system. After the flare and onset of the fast wind in 1987 July, the envelope was ejected. From that time PU Vul behaves as a typical very slow nova, with $\left[t_{2}\right]_{\mathrm{V}}=1016 \mathrm{~d}$ and $\left[t_{2}\right]_{\mathrm{B}}=1123 \mathrm{~d}$. Using the $M_{\mathrm{B}}-t_{2}$ relation, we have calculated $M_{\mathrm{B}}=-5.83$. The corresponding mass of the white dwarf, calculated using the relation of Livio (1992), is $M_{\mathrm{wd}}=0.57 \mathrm{M}_{\odot}$. Emission line profiles during the nebular stage of the nova (after 1991) show an expanding envelope in the form of an equatorial ring and polar blobs. The radial velocities of these substructures were used to determine the inclination angle of the inner orbit as $i=78^{\circ}$. Using the values of $i, M_{\mathrm{wd}}$ and the mass function $f\left(M_{\mathrm{wd}}, M_{\text {giant }}, \sin i\right)=1.6 \mathrm{M}_{\odot}$, we have determined the mass of the inner $\mathrm{M}$ giant as $M=2.6 \mathrm{M}_{\odot}$.

Analysis of photometric and spectroscopic data have revealed that the very slow, eclipsing symbiotic nova $\mathrm{PU}$ Vul is a hierarchical triple system consisting of a white dwarf $\left(M=0.57 \mathrm{M}_{\odot}\right)$ and a $\mathrm{M}$ giant $\left(M=2.6 \mathrm{M}_{\odot}\right)$ in an inner orbit $(P=761 \mathrm{~d})$, with the second $M$ giant in an outer orbit $(P=4882 \mathrm{~d})$. The mass ratio of the outer to inner orbit is $X=6.4$. Among the group of hierarchical triple systems that includes $\lambda$ Tau $(X=8.3)$ and $\mathrm{CH}$ Cyg $(X=7.0)$, PU Vul has the most extreme mass ratio.

\section{References}

Belyakina, T. S., Bondar, N. I., Chochol, D., et al., 1989, A\&A, 223, 119

Kanamitsu, O., Yamashita, Y., Norimoto, Y., et al., 1991, PASJ, 43, 523

Kolotilov, E. A., Munari U., Yudin B. F., 1995, MNRAS, 275, 185

Liller, M. H., Liller, W., 1979, AJ, 84, 1357

Livio, M., 1992, Ap. J., 393, 516

Nussbaumer, H., Vogel, M., 1996, A\&A, submitted

Stellingwerf, R. F., 1978, Ap. J., 224, 923

Vogel, M., Nussbaumer, H., 1992, A\&A, 259, 525 